\title{
Fabrication and characterization of sterically stabilized liposomes of topotecan
}

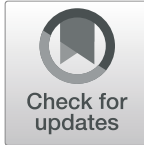

Dasharath Patel ${ }^{1}$ and Niteshkumar Patel ${ }^{2^{*}}$

\begin{abstract}
Background: Recently, the development of drug delivery which delivers controlled drug release at the tumor sites emerged as an attractive option for enhancing anticancer therapeutics. Next-generation nanotherapeutics must not contain only the nanoscale but should find their way to the solid tumor via active or passive targeting. Surface modification by pegylated lipids is one of the approaches used to made liposomes long-circulating and passively target the tumor. Pegylation of liposomes help them to alter the pharmacokinetics of drug molecule in vivo. The successful journey of such a complex drug delivery system from bench to clinic requires in-depth understanding and characterization. In this research, we fabricated and characterized sterically stabilized liposomes of topotecan which meets the clinical need. Liposomes have been prepared using ethanol injection-solvent evaporation method followed by extrusion for size reduction. Outer medium was replaced with an isotonic sucrose solution using dialysis followed by drug loading. We characterized liposomes' membrane phase and dynamics, drug and lipid quantification, size distribution, state of encapsulated drug, internal volume and internal pH of liposomes, presence, and thickness of grafted PEG on the liposomes surface, and in vitro leakage test.
\end{abstract}

Results: All these studied parameters directly or indirectly provide information regarding the pharmacokinetic behavior of the formulation and the tumor-targeting property of the drugs in vivo. We encapsulated the topotecan in nanoliposomes with pegylation on the surface resulting in long-circulating stealth liposomes. Nanoliposomes remotely loaded with topotecan by transmembrane gradient method.

Conclusion: Our in vitro characterization of topotecan liposomes provides an explanation for the good therapeutic efficacy of tumor cells.

Keywords: Topotecan, Sterically stabilized liposomes, Pegylated liposomes, Characterization, In vitro drug release, Remote loading

\section{Background}

Limitations of small molecule chemotherapeutics

The efficacy of currently available anticancer agents is mainly compromised due to their drawbacks like poor solubility, poor bioavailability, lack of selectivity for normal and cancer cells resulted in toxicity. After entering into the body, every molecule passes through the liver and undergoes metabolism by Cytochrome P450 and other enzymes [1]. This first-pass metabolism changes

\footnotetext{
* Correspondence: nitesh.patel98@gmail.com

${ }^{2}$ Department of Pharmaceutical Sciences, Hemchandracharya North Gujarat University, Patan, Gujarat, India

Full list of author information is available at the end of the article
}

the pharmacokinetic profile of the molecule by altering target specificity, solubility, and other physic-chemical parameters which make the drugs inactive or with modified toxicity and bioavailability profiles. Change in pharmacokinetic profile leads to a larger dose to maintain therapeutic concentration. Similarly, lengthy infusion may require to overcome changed solubility challenges. Due to metabolism changes, organs responsible for drug clearance face more damages due to changed properties; for example, cisplatin causes severe nephrotoxicity [2], and camptothecin regains activity in the acidic environment of the bladder to induce toxicity. Due to the nonselectivity of current chemotherapeutic agents, normal 
body cells that are in the growing stage like bone marrow and digestive tissues are getting affected and causing most side effects associate with anti-cancer agents. To overcome these side effects, one of the alternatives is to change the molecule to its salt form or slight modification in the chemical moiety. But this approach may lead to giving additional toxicities or diminished activity $[3,4]$. So, different delivery platforms like polymers, liposomes, and nanoparticles are being explored as effective methods to modulate drug activity [5-9]. Most of the large molecular carriers move drugs to the site of action, thereby limiting metabolism and reduce the toxicity. These carriers should have several favorable features including but not limited to water solubility, long circulation times with half-lives ranging from hours to days, lack of toxicity and immunogenicity, high drug loading capacity, and biocompatibility. As the pharmacokinetic property of drugs will be decided by the carrier system, longer circulation and high drug loading capacity can be achieved by changing the different physicochemical properties of the carrier system. Further, these carrier systems offer an advantage of passive targeting of a molecule to the tumor site by the mechanism known as enhanced permeation and retention effect (EPR). Endothelial cells of the tumor are having very poor vasculature due to high nutritional demand and rapid growth. There is very little or no lymphatic system available due to abnormal growth. This environment creates a favorable condition for novel nanocarriers to accumulate and target the encapsulated drugs at the site of the tumor. Normal tissue contains tight junctions between the blood capillary cells, so preventing macromolecules but small molecules to enter in normal tissue leads to poor tumor targeting and systemic toxicities.

\section{Rationale for selecting topotecan}

Topotecan, a semi-synthetic analog of camptothecin [10], is currently approved by the regulatory agency to treat refractory ovarian cancer and/or small-cell lung cancer, which are resistant to conventional chemotherapeutic agents [11, 12]. Furthermore, topotecan is also used in combination with other standard chemotherapeutic agents for improved therapy [13]. In line with other camptothecin derivatives, topotecan inhibits the activity of topoisomerase I, resulting in lethal DNA strand breaks. Although the excellent antineoplastic activity of topotecan, its clinical use is limited due to severe systemic toxicity. Preclinical studies revealed greater antitumor efficacy if given in low-dose exposure for prolong time resulted in reduced toxicity $[14,15]$. Therefore, improvement in antitumor efficacy and reduction in systemic toxicity could be achieved by maintaining prolonged exposure of topotecan to tumor tissues. Liposomes have been reported to prolong the exposure time of topotecan and other therapeutic agents in vivo $[16,17]$.
Another drawback associated with topotecan is its instability and conversion into inactive form carboxylate form by opening the lactone ring at physiological $\mathrm{pH}$. Liposomes can be used to encapsulate topotecan, which can bind to the phospholipids with different affinities. Further, upon encapsulation of topotecan, the inner environment of liposomes stabilizes the lactone form by preventing the hydrolysis in the physiological environment. Several researchers demonstrated improved stability of liposomeencapsulated topotecan as compared to free topotecan in vivo. However, usage of conventional passive loading techniques result in low encapsulation efficiency which limits the success of liposomal drug delivery. To overcome this limitation, different gradient loading methods have been used to entrap topotecan into the liposomes.

Liposomal drug delivery has been used for three decades to encapsulate anticancer agents to alter tissue disposition, reduce blood clearance rate, decrease systematic toxicity, and enhance antitumor activity $[18,19]$. In the case of topotecan, liposomal encapsulation could further provide the added advantage of protection against hydrolysis of the lactone ring. The closed lactone ring was reported to be structurally important for both passive diffusion of topotecan into cancer cells $[20,21]$ and successful interaction with the topoisomerase target [22].

Only the encapsulation of anticancer agents in liposomes might not be sufficient to achieve the target because of the RES uptake mechanism. Though nanoliposomes because of its EPR effect have been reported to enhance antitumor efficacy and reduce systematic toxicity, to make longcirculating liposomes, pegylation is mandatory. The thymus is a central immune organ, where lymphocytes mature, multiply, and become T cells [23]. Similarly, the spleen is a source of plasma cells and hence antibodies for the cellular and humoral-specific immune defenses [24]. Both organ play an important role in altering the pharmacokinetics of encapsulated liposomes. Pegylation helps liposomes to skip from phagocytosis and make liposomes long circulation which indirectly makes them passively targeting to tumor.

\section{Rationale for selecting ammonium phosphate as remote loading agent}

In the present study, ammonium phosphate is used as a remote loading agent for efficient loading of topotecan. To our surprise, we found that topotecan precipitates not only in the presence of sulfate ions, but also in the presence of phosphate ions. Topotecan was efficiently loaded into liposomes with a transmembrane $\left(\mathrm{NH}_{4}\right)\left(\mathrm{H}_{2} \mathrm{PO}_{4}\right)$ gradient. This phenomenon proposes a superior alternative technique of loading topotecan into lipid vesicles. Topotecan liposomes loaded with transmembrane $\left(\mathrm{NH}_{4}\right)\left(\mathrm{H}_{2} \mathrm{PO}_{4}\right)$ gradient show prolong circulation. This may improve the applicability of topotecan liposomes at tumor sites, which exhibit a decreased $\mathrm{pH}$ value compared with the non- 
tumor environment and keep intact form of lactone ring at the tumor site. Furthermore, this study helps to understand the loading mechanisms and encapsulation phenomenon of topotecan into liposomes. This method of encapsulation might be proved as a superior to other methods offering comparative therapeutic efficacy.

\section{Methods}

\section{Materials}

Hydrogenated soy phosphatidylcholine (HSPC) was obtained from Lipoid $\mathrm{GmbH}$. Cholesterol $(\mathrm{CH})$ was supplied by Dishman Netherland BV. 1,2-Distearoyl-sn-glycero-3-phosphoethanolamine-N-[methoxy(polyethylene glycol)-2000] (mPEG2000-DSPE) was purchased from Lipoid GmbH. Topotecan $\mathrm{HCl}$ (TPT) was purchased from Sigma. Oasis ${ }^{\circ}$ HLB 1 cc $(30 \mathrm{mg})$ extraction cartridge, was obtained from the Water incorporation, USA. All other chemicals were of analytical grade.

\section{Preparation of liposomes}

Sterically stabilized liposomes have been prepared using the ethanol injection solvent evaporation method. Lipid excipients containing HSPC/CH/ mPEG2000-DSPE (3/1/ $1, \mathrm{~W} / \mathrm{W}$ ) were dissolved in ethanol and injected into the solution containing a remote loading agent (ammonium phosphate, $250 \mathrm{mM}$ solution) at pre define temperature of $65^{\circ} \mathrm{C}$. Multilamellar vesicle (MLV) formed in the above step was introduced for size reduction using the extrusion technique (Lipex ${ }^{\oplus}$ by Northern lipids). The extrusion process was carried out at $65^{\circ} \mathrm{C}$ using four polycarbonate membranes of $80-\mathrm{nm}$ pore size at an operating pressure of 250 psi. Particle size distribution of formed small unilamellar liposome was measured using dynamic light scattering (Malvern zeta sizer ZS-90) technique.

\section{Preparation of the transmembrane ion gradient for drug encapsulation}

Liposomes were processed through an ultrafiltration assembly (with 750-Kd dialysis membrane made up of PES membrane). Extraliposomal ammonium phosphate solution was replaced with a $10 \%$ sucrose solution. The ultrafiltration process was continued until the conductivity of the permeate solution became equal to that of the conductivity of a $10 \%$ sucrose solution. Upon complete replacement of outer medium (ammonium phosphate solution) with the intended medium (10\% sucrose solution) for extra liposomal environment, the ultrafiltration process was ended and liposomal bulk was stored at $2-8^{\circ} \mathrm{C}$ till further processing.

\section{Encapsulation of topotecan inside the liposomes}

Empty liposomes prepared in the above step contained the inner medium of ammonium phosphate while the outer medium contained a 10\% sucrose solution. To load the empty liposomes with topotecan, an empty liposomal solution was kept above glass transition $(\mathrm{Tg})$ temperature $\left(58^{\circ} \mathrm{C}\right)$ under stirring. Topotecan hydrochloride was added in empty liposomal dispersion to get the final concentration of topotecan $1 \mathrm{mg} / \mathrm{mL}$ under stirring. The dispersion was incubated to load the drug inside liposomes with maintaining the temperature of dispersion above $\mathrm{Tg}$ for a minimum of $60 \mathrm{~min}$ followed by cooling of dispersion at a temperature between 2 and $8{ }^{\circ} \mathrm{C}$.

\section{Drug and lipid quantification}

Drug quantification was done as per the method described in [25]. The system included Waters HPLC autosampler and Waters data system. Topotecan was quantified using a Waters Symmetry C18 column (150 $\mathrm{mm} \times 4.6 \mathrm{~mm}, 5 \mu \mathrm{m})$ with a fluorescence detector (Jasco Model FP-210) at excitation/emission wavelengths of $416 / 522 \mathrm{~nm}$. The mobile phase consists of water (with $0.6 \%$ acetic acid and $1.5 \%$ triethylamine) and acetonitrile $(82.5: 17.5, \mathrm{v} / \mathrm{v})$. The analytical method was validated for its precision, limit of detection (LOD) and limit of quantification (LOQ), linearity, accuracy, robustness, and solution stability.

Lipid quantification was performed using HPLC with Charged Aerosol Detector. All three lipids (HSPC, Cholesterol, and mPEG-DSPE-2000) were quantified using a Durashell C18 (L), $150 \mathrm{~mm} \times 4.6 \mathrm{~mm}, 5 \mu \mathrm{m}, 150 \mathrm{~A}^{\circ}$ with a CAD detector (Dionex). The mobile phase consists of water/methanol (with ammonium acetate). Methanol was used as a diluent to prepare samples. The analytical method was validated for its precision, limit of detection (LOD) and limit of quantification (LOQ), linearity, accuracy, robustness, and solution stability.

\section{Encapsulation efficiency}

Encapsulation efficiency was measured by subtracting the free topotecan from the total topotecan. Free topotecan content in the liposomal dispersion was measured after separation. Separation of free topotecan was achieved using a solid-phase extraction cartridge (Oasis ${ }^{\circ}$ HLB $1 \mathrm{cc}(30 \mathrm{mg})$ extraction cartridge). The quantification of separated free drugs was measured as described in the previous section. Encapsulated topotecan was calculated using the formula:

$$
\% \text { Entraped topotecan }=\frac{(\% \text { content of total topotecan }- \text { content of } \% \text { free topotecan }) * 100}{\% \text { content of total topotecan }}
$$




\section{State of encapsulated drug inside topotecan-loaded liposomes}

Various techniques were used for the determination of the physical form of drug present inside the liposomes. Fluorescence studies, X-ray diffraction (small angle), and Cryo-TEM were used to determine the state of encapsulated drugs.

Fluorescence measurements were done using a Cary Eclipse fluorescence spectrophotometer, equipped with a $400 \mathrm{~W}$ Xe lamp as an excitation source. Three samples were evaluated (drug substance, placebo liposomes, and topotecan-entrapped liposomes) for fluorescence studies.

A small-angle X-ray study was performed using Xray Generator: Micro-focus rotating anode and SAXS Model \& Make: NANO-Viewer, Rigaku, Japan, SAXS Detector: 2D Hybrid Pixel Array Detector. For a small angle $\mathrm{X}$-ray study, the liposomal samples were centrifuged at about $60,000 \mathrm{rpm}$ for $6 \mathrm{~h}$ at $4{ }^{\circ} \mathrm{C}$ to get pellets of vesicles, dried in air to remove excess water. The pelleted samples, which looked like gels, were applied on transparent tape and covered with the same tape before fitting into the sample holder. The sample holder was then put into the vacuum chamber, the vacuum was created inside the chamber, and the position of the samples ( $\mathrm{X}$ and $\mathrm{Y}$ direction) was identified with the help of a nanograph. Each sample was scanned for $1 \mathrm{~h}$ under X-ray. Scattering from a $10 \%$ sucrose solution was also collected for $1 \mathrm{~h}$ and later subtracted from the profile of individual samples to subtract the background. Scattering profile of samples was pre-computed and integrated from $2 \mathrm{D}$ image to generate the histogram of intensity vs scattering profile.

Three concurrent and prominent peaks in the resultant histogram of all samples are manually picked up using Origin Pro 8 data analysis package. The data was analyzed for intensity vs $2 \theta$, where $\theta$ (theta) is the angle of scattering. The data was also analyzed for Lorentz correction and intensity vs $q$, where $q$ is the scattering vector.

Cryo TEM analysis was performed by a frozenhydrated vitrified technique using a semi-automated system Vitrobot Mark IV. About $4 \mu \mathrm{l}$ of liquid sample was taken on a holey fomvar carbon film 200 mesh $\mathrm{Cu}$ (Cu-200HFC Pacific Grid Tech www.grid tech.com) and transferred to a Gatan model 655 cryo holder with a cryo-transfer system and then cryo-transferred into the TEM goniometer while maintaining the cold chain throughout. Imaging was done in a JEOL 2100 HRTEM operating at $200 \mathrm{KeV}$ while maintaining the sample holder at about - 172 to $-174{ }^{\circ} \mathrm{C}$ as measured by a Gatan Smartset model 900 cold stage controller. Images grabbed by Orius
Camera Gatan make controlled by digital micrograph software.

\section{Internal environment of topotecan-encapsulated liposomes}

The assessment of internal volume and internal $\mathrm{pH}$ were fulfilled by performing studies like internal volume measured by Cryo-TEM and internal $\mathrm{pH}$ of liposomes using epi-fluorescence microscopy. Data obtained in the previous section of Cryo-TEM was utilized to calculate the internal volume of placebo liposomes and drugloaded liposomes. The internal volume of liposome was measured using the formula, Volume $=\frac{4}{3} \pi r^{3}$ where $r$ was derived from subtracting bilayer thickness from mean particle size.

To measure internal $\mathrm{pH}$, compound (pyranine) having $\mathrm{pH}$-dependent fluorescent property was selected. The solution of pyranine was incubated with placebo liposomal dispersion and drug-loaded liposomal dispersion. The outer medium was replaced using the ultrafiltration process with a $10 \%$ sucrose solution. Fluorescent intensity of drug-loaded liposomes and placebo liposomes was measured and compared with a standard calibration curve of fluorescent spectra obtained with different $\mathrm{pH}$ solutions.

\section{Liposome surface property, bilayer property, and size distribution}

Surface morphology of liposome was studied using atomic force microscopy. AFM analysis is done using a Veeco multimode scanning probe microscope with a nanoscope IV controller. The data were acquired and processed using the Nanoscope software, version 6.12r2. All the AFM measurements were done under ambient conditions using the tapping-mode AFM probes model Tap190Al. Samples were dispersed in distilled water (1 $\mathrm{mg} / 2 \mathrm{~mL}$ ). Silicon wafer was cleaned in distilled water and piranha solution. The diluted aqueous solution was drop cast on a cleaned silicon wafer and allowed to dry for AFM study.

Liposome bilayer property was studied using differential scanning calorimetry. DSC study was performed using a Q200 differential scanning calorimeter of TA instruments. The liposomal samples were centrifuged at $50,000 \mathrm{rpm}$ for $4 \mathrm{~h}$ at $4{ }^{\circ} \mathrm{C}$. The liposomal pellet was thus obtained, was then weighed, and kept in an aluminum crucible and heated at elevated temperature. Liposomal sample without drug was scanned in the temperature range from -20 to $100{ }^{\circ} \mathrm{C}$ at a heating rate of $5{ }^{\circ} \mathrm{C} / \mathrm{min}$ whereas the drug-loaded liposomes were scanned at a range from $10-100{ }^{\circ} \mathrm{C}$ with the same heating rate of $5^{\circ} \mathrm{C} / \mathrm{min}$. 
Particle size distribution was measured using the dynamic light scattering technique (Nano Brook 90 Plus, Brook haven instrument). Analysis was performed by diluting samples to 10 times with water and measuring at a $90^{\circ}$ angle.

\section{Measurement of PEG layer thickness on sterically stabilized liposomes}

The surface modification with methoxy polyethylene glycol (MPEG) polymer prevents the liposomes from rapid clearance by RES uptake and increases blood circulation time. The thickness of the pegylation layer should be thermodynamically limited and in several nanometers. The evaluation of the PEG layer was completed by measuring fixed aqueous layer thickness as described in reference [26]. Increment in salt concentration will affect the zeta potential, and this relationship was used to derive PEG layer thickness as a function of slop.

\section{Electrical surface potential or surface charge}

Surface charge or zeta potential was measured by the Zeta sizer (Malvern instrument, Nano ZS-90 model) using Clear disposable zeta cell.

\section{In vitro leakage of topotecan from liposomes}

In vitro drug leakage testing to characterize the physical state of the lipid bilayer and encapsulated topotecan should be investigated to support a lack of uncontrolled leakage under a range of physiological conditions and equivalent drug delivery to the tumor cells [27]. Topotecan liposomes were studied for in vitro drug leakage at $37^{\circ} \mathrm{C}$ in $50 \%$ human plasma for $24 \mathrm{~h}$. As per method, study was performed using 50\% human plasma and $\mathrm{K}_{2}$ EDTA was used as an anticoagulant. The analytical technique used was LC-MS/MS with a mass spectrometer as a detection mode. Solid-phase extraction was used to determine free topotecan while protein precipitation technique was used to determine total topotecan inside the plasma sample. In chromatographic and mass spectrometric system, Hypurity $\mathrm{C}_{18}(50 \times 4.6 \mathrm{~mm}) 5 \mu \mathrm{m}$ column was used with Acetonitrile(Pump-A): Buffer (Pump-B) (35:65, respectively) as mobile phase and acetonitrile: water $(80: 20, \mathrm{v} / \mathrm{v})$ as rinsing solution. In mass parameters (API-4500 QTrap), ion source was ESI, and polarity was kept positive. \% Leakage of topotecan was measured using the following formula.
In vitro release of topotecan from liposomes at different conditions

In vitro drug release testing to characterize the release behavior encapsulated topotecan should be investigated to support the prediction of release of the drug under a range of physiological conditions and equivalent drug delivery to the tumor cells. Topotecan liposomes were studied for in vitro drug release at different conditions like $\mathrm{pH}=7.5$, 6.5, and 5.5 at $37^{\circ} \mathrm{C}$ and at $43{ }^{\circ} \mathrm{C}$ and $57^{\circ} \mathrm{C}$ at $\mathrm{pH}=6.5$ for $24 \mathrm{~h}$. As per method, study was performed using Bottle rotating apparatus with a Water bath heating system (Make: Electrolab, Model: ERB-16 W) with 100-mL capacity glass bottles with a screw cap. The sample volume was $1 \mathrm{~mL}$, and medium volume was $60 \mathrm{~mL}$. Free drug released from liposome was trapped using Dowex 50 WX4 hydrogen form (200-400 mesh). So supernatant provide encapsulated drug. Subtraction of encapsulated drug from total drug (\% assay) provides \% release drug quantity. Quantification of topotecan was performed as per the details mentioned in section encapsulation efficiency.

\section{In vitro cell line study (MTT assay)}

The MTT assay was used as a measure of the antitumor activity of topotecan liposomes. In brief, $100 \mathrm{~mL}$ of cell suspension containing the required cell number was added to the wells of 96-well plates and incubated at $37^{\circ} \mathrm{C}$ in humidified air with $5 \% \mathrm{CO} 2$. After $24 \mathrm{~h}, 100 \mathrm{~mL}$ of cell culture medium containing the appropriate concentration of topotecan hydrochloride or their liposomal formulations in the desired concentration range was added to these cells. After $72 \mathrm{~h}, 50 \mathrm{~mL}$ of MTT solution was added to the wells, and plates were incubated at $37^{\circ} \mathrm{C}$ for $3.5 \mathrm{~h}$. Supernatant was aspirated before dissolving the formazan crystals in $150 \mathrm{~mL}$ dimethyl sulfoxide (DMSO). Plates were agitated for 10 min, and the optical density of each well was read at 570 $\mathrm{nm}$ using a microplate reader. Cytotoxicity was measured by comparing absorbance from treated wells against that from control wells (cells treated with culture medium instead of drug). Concentrations of topotecan or its liposomal formulation needed to cause a $50 \%$ loss in viability as judged by the MTT assay (IC50) were determined.

\section{Results \\ Drug and lipid quantification}

Drug and lipids quantification was measured using the method described in the material and method section. Summarized results are tabulated in Table 1.

$$
\% \text { Leakage at each time point }=\frac{(\text { Free drug at respective time point }- \text { Free drug at } 0 \text { time }) * 100}{\text { Entrapped drug at } 0 \text { time }}
$$


Table 1 Quantification results of drug and lipids in topotecan liposomes

\begin{tabular}{lll}
\hline Ingredients & $\begin{array}{l}\text { Labeled amount } \\
\text { in } \mathbf{~ m g / m L}\end{array}$ & $\begin{array}{l}\text { Results } \\
\text { Value in } \% \pm \text { SD }(\boldsymbol{n}=\mathbf{3})\end{array}$ \\
\hline Topotecan & 1.00 & $100.3 \pm 1.3$ \\
MPEG 2000-DSPE & 3.19 & $99.8 \pm 2.1$ \\
Sodium salt (Lipoid) & & $101.6 \pm 1.9$ \\
HSPC (Lipoid S PC-3) & 9.58 & $100.6 \pm 0.3$ \\
Cholesterol & 3.19 & \\
\hline
\end{tabular}

The complete composition of the formulation is presented in Table 2.

\section{Determination of encapsulation efficiency}

$\%$ Free drug found in this formulation was $0.64 \%$. Using equation mentioned in the "Methods" section, calculated encapsulation efficiency in present formulation found to be $99.36 \pm 0.18(n=3)$ when drug loading was done at $65 \pm 5^{\circ} \mathrm{C}$ for $60 \mathrm{~min}$ followed by cooling at $8^{\circ} \mathrm{C}$ for 30 min under stirring.

\section{State of encapsulated drug inside topotecan-loaded liposomes}

As discussed in the material and method section, three different techniques adopted to characterize the state of encapsulated drug which are as follow.

1. Fluorescence studies

2. X-ray diffraction (small angle)

3. Cryo-TEM

\section{Fluorescence studies}

Fluorescence study has been done to observe the emission peak of the samples. Fluorescence emission spectra of topotecan drug substance and placebo liposome injection were recorded at $\lambda_{\mathrm{ex}}=470 \mathrm{~nm}$. Upon excitation at $\lambda_{\text {ex }}=470 \mathrm{~nm}$, an emission peak at $588 \mathrm{~nm}$ is observed for the drug substance, while fluorescence emission was

Table 2 Composition of topotecan liposome formulation

\begin{tabular}{ll}
\hline Ingredients & $\begin{array}{l}\text { Labeled amount } \\
\text { in } \mathbf{~} \mathbf{g} / \mathbf{m L}\end{array}$ \\
\hline Topotecan & 1.00 \\
MPEG 2000-DSPE & 3.19 \\
Sodium salt (Lipoid) & \\
HSPC (Lipoid S PC-3) & 9.58 \\
Cholesterol & 3.19 \\
Sucrose & 100 \\
Ammonium phosphate* & - \\
Water for injection & q.S to $1 \mathrm{~mL}$ \\
\hline *Used to create ammonium gradient and removed during processing with \\
traces remaining
\end{tabular}

NOT observed in the placebo batch. Upon excitation at $\lambda_{\text {ex }}=470 \mathrm{~nm}$, two emission peaks are observed for all the samples of topotecan liposomes. The spectra were recorded in the range of $500-800 \mathrm{~nm}$, and the peaks were observed in the range of 55 to $700 \mathrm{~nm}$. Refer to Table 3.

The figure for fluorescence spectra of topotecan drug substance, placebo liposome, and Topotecan liposome are given in Fig. 1.

The fluorescence emission spectra of topotecan liposome are considerably quenched as compared to significantly intensified emission of drug substance which is indicative of the encapsulation of topotecan inside the liposome.

\section{$X$-ray diffraction study}

Results obtained during the SAXS study are tabulated in Table 4.

The demanding sample treatment is accountable for differences in peak intensity across the samples provided a resemblance of peaks appearance across the samples. Figures 2 and 3 show that the SAXS peak (2 theta)-1 was observed at about 1.1 while peaks 2 and 3 were marked at about 1.6 and 3.4 respectively in the topotecan liposome which is absent in placebo. This indicates the precipitation of topotecan inside the liposome which helps to sustain release of drug when introduced in the body.

\section{Cryo-transmission electron microscopy (Cryo-TEM)}

The objective of the study is to evaluate the CryoTEM analysis of topotecan liposome with placebo liposome. Cryo-TEM is the technique used to get the information about the internal structure and internal environment of the liposome. In order to achieve a greater understanding of the interactions between topotecan and ammonium phosphate, this technique has been used. Cryo-TEM analysis of topotecan liposome and placebo liposome is discussed and presented in Tables 5 and 6.

Results revealed about 100\% sphericity and \% unilamellarity observed in topotecan liposome and placebo

Table 3 Observation of fluorescence study for drug substance, placebo liposome, and drug-loaded liposome of topotecan

\begin{tabular}{lll}
\hline Sample details & Wavelength & \\
\cline { 2 - 3 } & $\begin{array}{l}\text { Emission } \\
\text { peak-1 }\end{array}$ & $\begin{array}{l}\text { Emission } \\
\text { peak-2 }\end{array}$ \\
\hline $\begin{array}{l}\text { Topotecan hydrochloride } \\
\text { drug substance }\end{array}$ & $588 \mathrm{~nm}$ & Not observed \\
$\begin{array}{l}\text { Placebo of topotecan } \\
\text { liposome }\end{array}$ & No peak & No peak \\
$\begin{array}{l}\text { Topotecan liposome } \\
\text { Toln }\end{array}$ & $558 \mathrm{~nm}$ & $598 \mathrm{~nm}$ \\
\hline
\end{tabular}



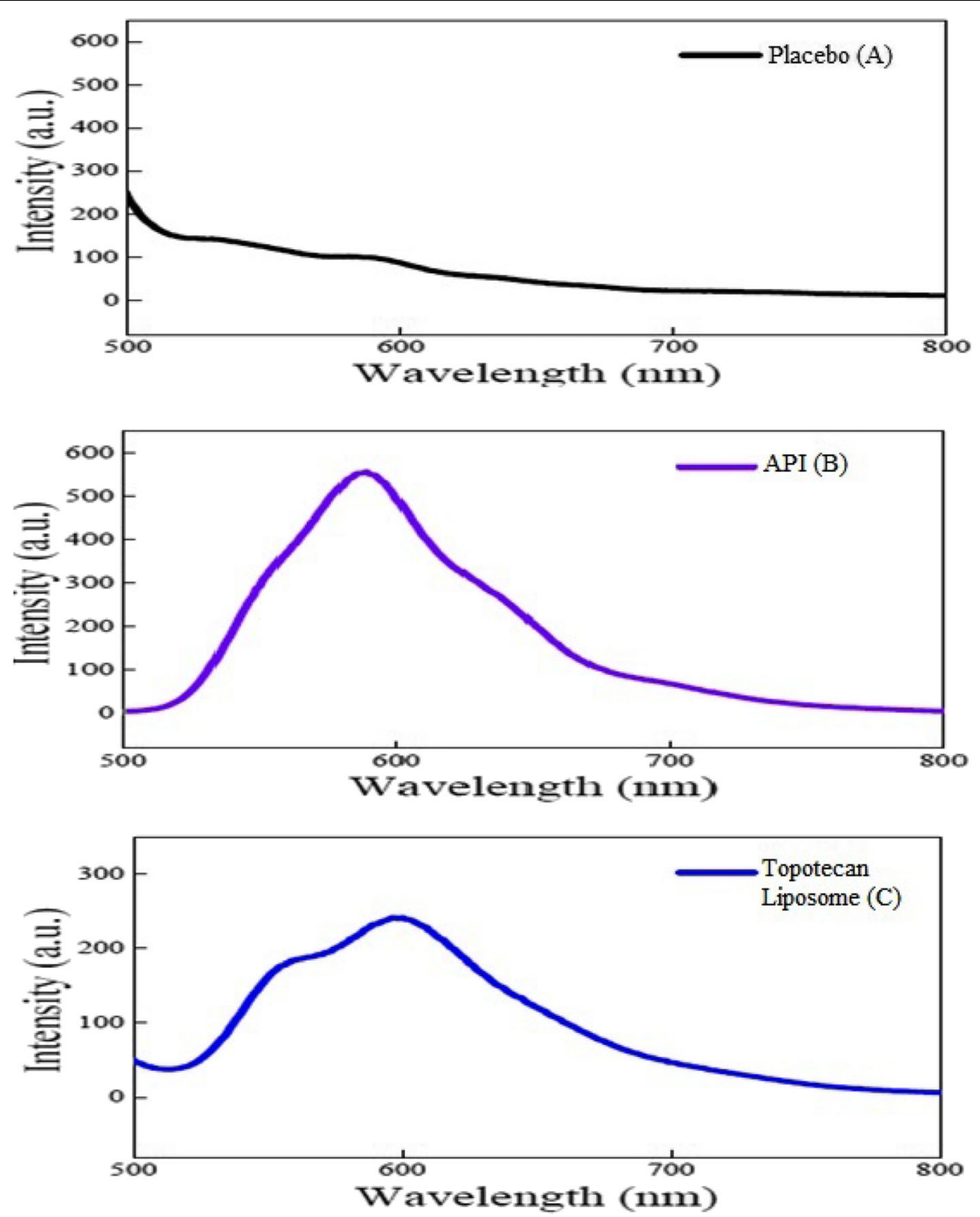

Fig. 1 a Fluorescence spectra of placebo. b Fluorescence spectra of API. c Fluorescence spectra of topotecan liposome

liposome. No any oblong or de-shaped liposomes were observed in Cryo-TEM analysis. The shape of liposomes before and after drug loading remains the same (spheres) which is a good indication with respect to optimized parameters of drug loading.

Some additional information was also derived during Cryo-TEM analysis. The liposomal internal volume observed in topotecan liposome and placebo liposome is about $4.0 \times 10^{4} \mathrm{~nm}^{3}$ to $5.8 \times 10^{4} \mathrm{~nm}^{3}$. The bilayer

Table 4 Concurrent and prominent SAXS peaks (2 theta)

\begin{tabular}{llll}
\hline Product & Peak I & Peak II & Peak III \\
\hline Topotecan liposome & 1.1 & 1.6 & 3.4 \\
Placebo of topotecan liposome & $\mathrm{Nil}$ & $\mathrm{Nil}$ & $\mathrm{Nil}$ \\
\hline
\end{tabular}

thickness (lamellar thickness) observed in topotecan liposome and placebo liposome is in the range of 4.6 to $7.1 \mathrm{~nm}$. Here, bilayer thickness means the thickness of phospholipid, a component of liposome which forms lipid vesicle. The internal volume of liposome was measured using the formula, Volume $=\frac{4}{3} \pi r^{3}$ where $r$ was derived from subtracting bilayer thickness from mean particle size. Bilayer thickness was measured using a calibrated scale of digital micrograph software.

The topotecan drugs are observed to be present inside the aqueous compartment of the unilamellar vesicles in the form of precipitates (needle shape) while the placebo does not contain any crystalline structure inside an aqueous compartment. 

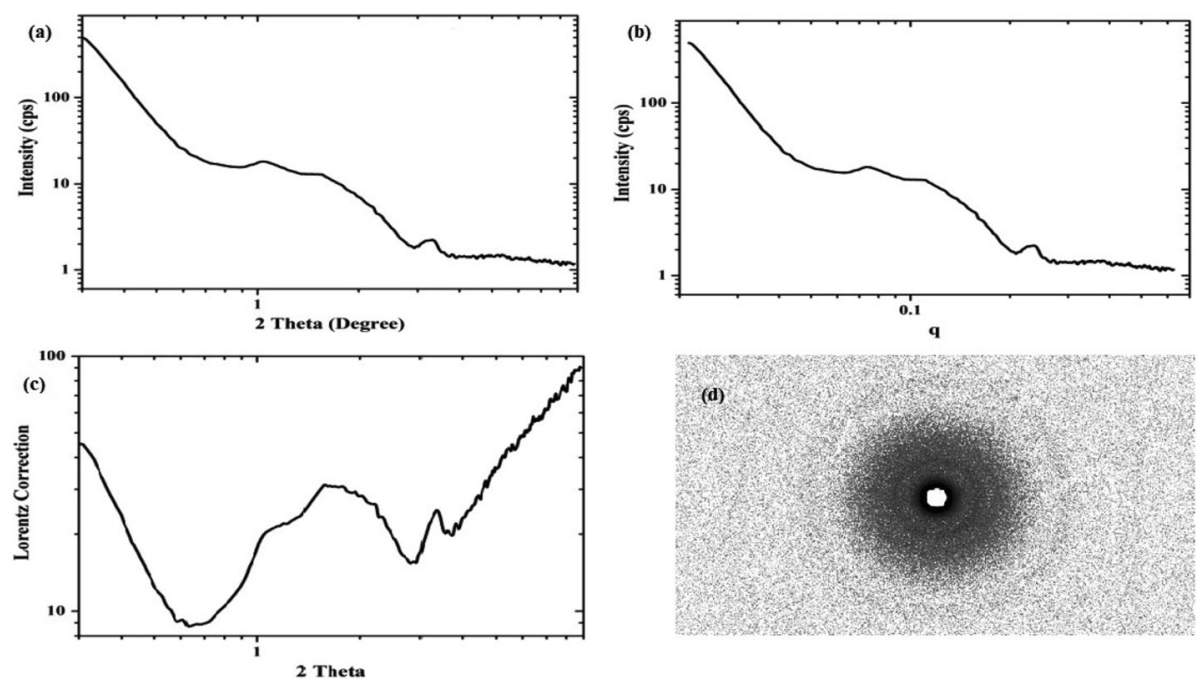

Fig. 2 Small angle X-ray scattering profile of topotecan liposome. a Intensity vs. 2 theta. b Intensity vs. q. c Lorentz correction. $\mathbf{d} 2 \mathrm{D}$ small-angle $X$-ray scattering profile

Cryo-TEM images of topotecan liposome and placebo liposome are presented as Figs. 4 and 5, respectively.

\section{Internal environment of topotecan-encapsulated liposome Internal volume of liposome}

The task has been accomplished by conducting CryoTEM studies. The said studies have been comprehensively discussed in the earlier section.

The findings for internal volume are discussed in Table 7.

The Cryo-transmission electron microscopic study reveals that the liposomal internal volume in topotecan liposome and placebo liposome is comparable and is in the range of 40,914 to $54,291 \mathrm{~nm}^{3}$.

\section{Internal $\mathrm{pH}$ of liposome}

The internal $\mathrm{pH}$ of topotecan liposome and placebo liposome were measured. The summary of the results is presented in Table 8.

All topotecan liposome and placebo liposome shows mean fluorescence intensity between 99 and $123 \mathrm{AU}$ which indicates that all the samples have an internal $\mathrm{pH}$ below 4.0.

From the above results, it can be concluded that liposome contains acidic pH (below 4.0) inside the liposome
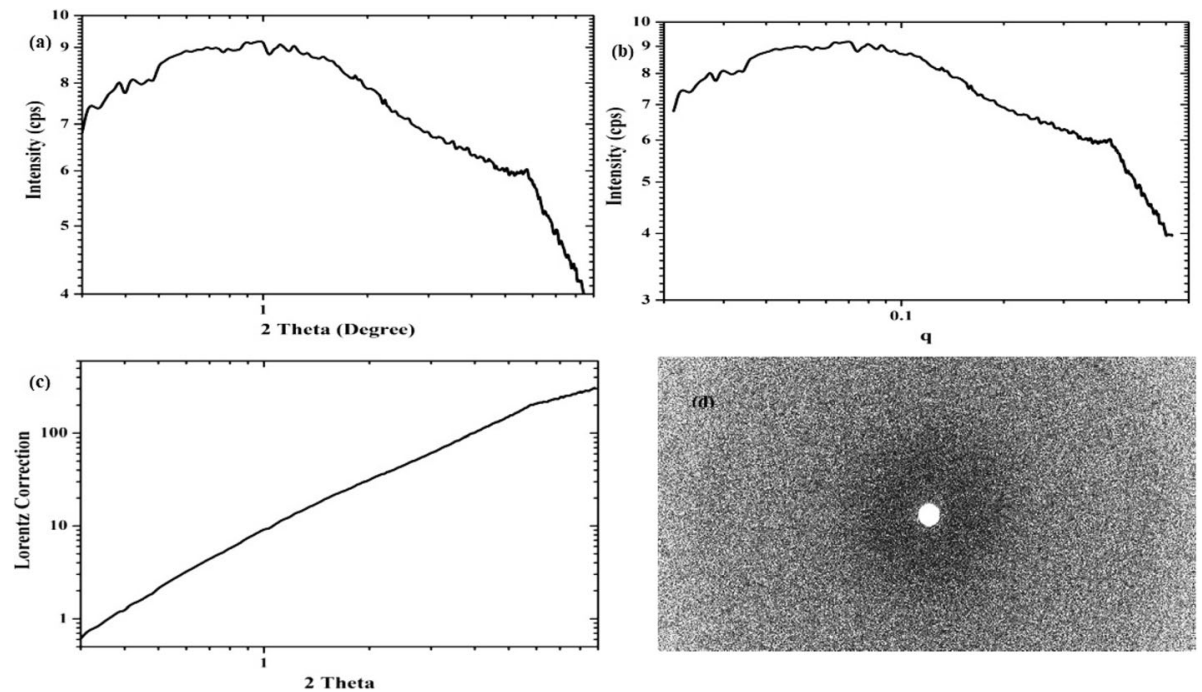

Fig. 3 Small angle X-ray scattering profile of placebo liposome. a Intensity vs. 2 theta. b Intensity vs. q. c Lorentz correction. $\mathbf{d} 2 \mathrm{D}$ small-angle Xray scattering profile 
Table 5 Cryo-TEM results for topotecan liposome and placebo liposome

\begin{tabular}{lllllll}
\hline Product name & Particle size & & Lamellarity & & $\begin{array}{l}\text { Drug } \\
\text { strand } \\
\text { thickness }\end{array}$ & $\begin{array}{l}\text { Number of } \\
\text { liposome } \\
\text { observed }\end{array}$ \\
\cline { 2 - 6 } Mean SD & Max & Min & & Unilamellar & $15.14 \pm 1.3$ & 301 \\
\hline Topotecan liposome & $51.9 \pm 7.8$ & 90.0 & 30.7 & Unilamellar & $17.8 \pm 0.9$ & 296 \\
Placebo liposome & $58.3 \pm 8.3$ & 89.9 & 34.0 & & &
\end{tabular}

which ensures an active and stable form of topotecan inside the liposome.

\section{Liposome surface property, bilayer property, and size distribution}

Liposome morphology should be determined as the morphology of liposome is key importance for the effective clinical translation of liposomal formulations. It provides the information regarding aggregation behavior which might affect the stability of the formulation.

Morphology of liposome is a critical aspect of the liposomal formulation. The morphology of liposome in topotecan liposome has been studied. The task has been completed employing the following studies.

Morphology of liposome by atomic force microscopy (AFM) The atomic force microscopic analysis of topotecan liposome and placebo liposome is tabulated Table 9 and presented in Fig. 6:

\section{Lipid bilayer property}

Differential Scanning calorimetry has been conducted to determine the lipid bilayer phase transition temperature.

In the differential scanning calorimetry curve, result revealed midpoint of glass transition was observed 58.88 ${ }^{\circ} \mathrm{C}$ for placebo liposome while $72.74{ }^{\circ} \mathrm{C}$ for topotecanloaded liposome. These differences confirmed internal changes and encapsulation of topotecan inside the liposome. Results are listed in Table 10.

Differential scanning calorimetry curves of the topotecan liposome and placebo liposome are presented in Fig. 7.

\section{Liposome size distribution}

The analysis has been carried out using dynamic light scattering (DLS) technique using Brook Haven Instruments (Model: 90 Plus).

The analysis has been carried out by dynamic light scattering (DLS) technique using Brook Haven Instruments (Model: 90 Plus) each of the topotecan liposomes and placebo liposomes, and results are tabulated in Table 11, and histogram are presented in Fig. 8.

Liposome size distribution found uniform in topotecan liposome and placebo liposome. The results for $D_{10}, D_{50}$, $\mathrm{D}_{90}$, Z-Average, PDI (Polydispersity index), and SPAN for all topotecan liposome and placebo liposome are comparable and indicate the uniformity of size distribution. There will not be any increase or decrease in size observed after drug loading inside the liposome.

Results obtained for size determined by these techniques are in concurrence with that obtained by Cryo-TEM analysis.

\section{Measurement of PEG layer thickness on sterically stabilized liposome}

The evaluation of the PEG layer has been accomplished employing fixed aqueous layer thickness measurement, and the results are tabulated in Table 12.

The results indicate that sufficient $\mathrm{nm}$ of pegylation on the surface of liposome ensures the long circulation inside the body.

\section{Electrical surface potential or surface charge}

Zeta potential has been determined by using Zeta sizer (Make: Malvern, Model: Nano ZS-90) at $25^{\circ} \mathrm{C}$. The results are tabulated in Table 13.

The results for zeta potential indicates that the effective surface charge on liposome make them thermodynamically stable due to colloidal property. They will remain in Brownian motion. These values indicate the stability of formed liposomes.

\section{In vitro leakage of topotecan from liposome}

In vitro drug leakage at $37^{\circ} \mathrm{C}$ in $50 \%$ human plasma for $24 \mathrm{~h}$ is used to study the release behavior of entrapped topotecan from sterically stabilized liposome. Free topotecan was measured from 50\% human plasma at 10 times dilution at different time point till $24 \mathrm{~h}$. Based on

Table 6 Summary of findings in Cryo-TEM analysis

\begin{tabular}{|c|c|c|c|c|c|c|c|c|c|}
\hline \multirow[t]{2}{*}{$\begin{array}{l}\text { Sr. } \\
\text { no. }\end{array}$} & \multirow[t]{2}{*}{ Product name } & \multirow[t]{2}{*}{$\begin{array}{l}\text { Group avg. internal } \\
\text { volume }\left(\mathrm{nm}^{3}\right) \pm S D\end{array}$} & \multirow[t]{2}{*}{$\begin{array}{l}\text { \% of } \\
\text { sphere }\end{array}$} & \multirow[t]{2}{*}{$\begin{array}{l}\% \text { of } \\
\text { ellipsoid }\end{array}$} & \multirow[t]{2}{*}{$\begin{array}{l}\text { Lamellar } \\
\text { thickness } \\
(\mathrm{nm})\end{array}$} & \multicolumn{3}{|c|}{$\begin{array}{l}\text { Drug strand thickness } \\
n=300 \text { liposomes (nm) }\end{array}$} & \multirow[t]{2}{*}{ Unilamellarity } \\
\hline & & & & & & Avg. & Max. & Min. & \\
\hline 1 & Topotecan liposome & $40,914 \pm 2348$ & 100 & 0 & 4.6 & $12.4 \pm 0.5$ & 26.0 & 7.1 & About 100\% \\
\hline 2 & Placebo liposome & $54,291 \pm 2829$ & 100 & 0 & 5.3 & NA & NA & NA & About 100\% \\
\hline
\end{tabular}



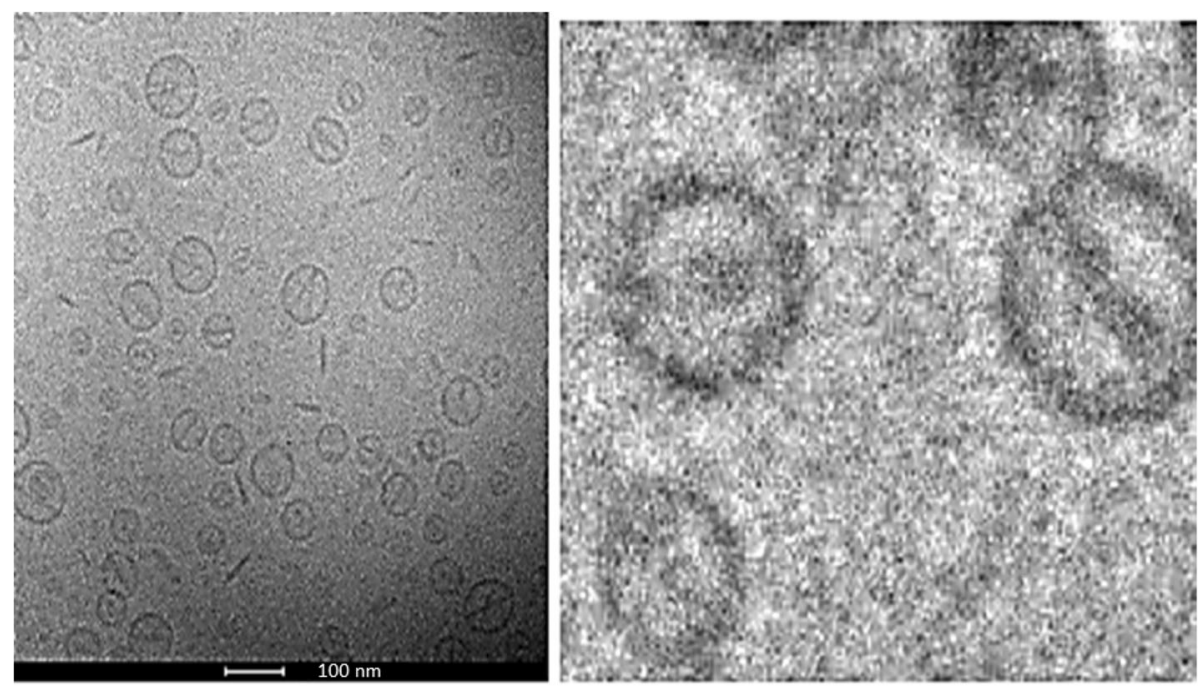

Fig. 4 Cryo-TEM images of topotecan liposome

the above parameters, \% leakage was calculated and is tabulated in Table 14 and graphically presented in Fig. 9.

The in vitro leakage of topotecan in 50\% human plasma when incubated at $37^{\circ} \mathrm{C}$ was studied at $200 \mu \mathrm{g} / \mathrm{mL}$ (10 times dilution) till $24 \mathrm{~h}$. The \% leakage with $200 \mu \mathrm{g} / \mathrm{mL}$ (10 times dilution), was found to be $\sim 1 \%$ till $24 \mathrm{~h}$. The results show that liposomal formulation is having long circulation and slow-release characteristics which help them to be long-circulating inside the body and passively targeting the tumor with desired concentration.

\section{In vitro release of topotecan from liposomes at different conditions}

Figure 10 summarizes the in vitro release behavior of topotecan from liposomes at different conditions that mimic the physiological environment. In vitro release is different than in vitro leakage. In vitro release was checked in the condition which was intentionally created a similar environment as inside the cancer cell (like lower $\mathrm{pH}$ up to 5.5 and higher temperature). The purpose to study in vitro release at different conditions was to predict the behavior of drug release inside the cancer cell environment in vivo, while the purpose of in vitro drug leakage studies in 50\% human plasma was to predict the leakage of topotecan from liposome when it gets normal physiological condition. Data from Fig. 10 show that the liposomes release topotecan very fast at $57^{\circ} \mathrm{C}$ while liposomes release topotecan slow at physiological temperature, i.e., $37^{\circ} \mathrm{C}$. Data revealed that liposome will release drugs faster while coming in contact with cancer cells which is having a somewhat higher temperature
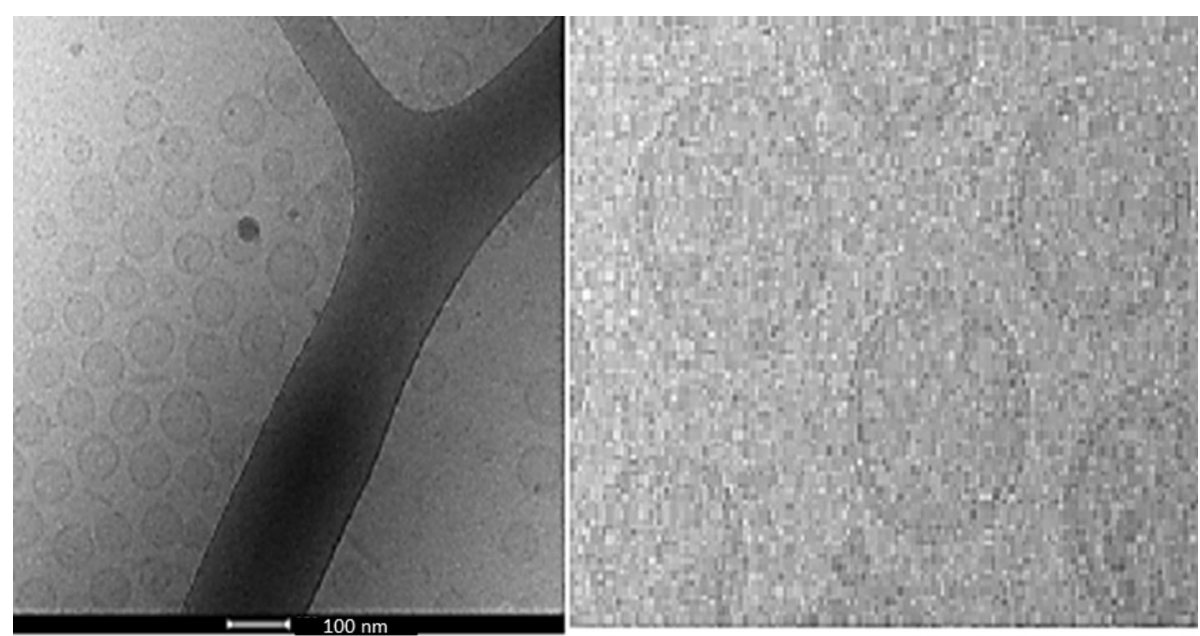

Fig. 5 Cryo-TEM images of placebo liposome 
Table 7 Internal volume results of topotecan liposome and placebo liposome

\begin{tabular}{lll}
\hline Sample details & Topotecan liposome & Placebo liposome \\
\hline No. of liposomes measured & 296 & 300 \\
Group Avg. internal volume $\left(\mathrm{nm}^{3}\right) \pm$ SD & $40,914 \pm 2348$ & $54,291 \pm 2829$
\end{tabular}

than normal physiological condition $\left(37^{\circ} \mathrm{C}\right)$. Similarly, the topotecan release rate was found faster in lower $\mathrm{pH}$ conditions which is expected to be inside the tumor cell. In vitro drug release study and in vitro drug leakage in $50 \%$ human plasma study revealed that topotecan liposome is expected to remain in long circulation mode in normal plasma and expected to release the drug faster when comes in contact in the tumor environment.

\section{In vitro cell line study (MTT assay)}

The MTT assay was used as a measure of cell viability. It is indirectly indicative of the cytotoxic efficacy of the formulation. The results obtained are depicted in Fig. 11.

Study shows lower inhibitory concentration is required for topotecan liposome as compared to control and blank liposome which indicate positive response in selected cell line. MTT assay determines the concentration required to kill $50 \%$ of the cancerous cell population. The strength of plain drug solution and liposomeencapsulated solution was the same during the study of MTT assay. Our objective for MTT assay was to check whether the encapsulation of topotecan inside the liposome compromises the cytotoxicity on cancerous cells or not. MTT results revealed there was no compromise of the cytotoxic property of topotecan on cancerous cells after encapsulation inside the liposomes.

Encapsulation will provide the in vivo advantage by reducing systemic toxicity to healthy cells and thus improve the safety profile of a molecule without any compromise in cytotoxicity on cancerous cells which is also supported by data of in vitro leakage study in $50 \%$ human plasma.

\section{Discussion}

The need to develop chemically and physically stable nanoliposomes, in which drug is encapsulated at a specific predefined mole ratio, requires an optimization of the loading conditions. The behavior of topotecan is strongly $\mathrm{pH}$-dependent due to its amphipathic nature. When the $\mathrm{pH}$ decreases below the $\mathrm{pKa}$ of these drugs, their amino group becomes protonated. This

Table 8 Summary of internal pH determination by UV intensity using pyranine

\begin{tabular}{lll}
\hline Product details & Topotecan liposome & Placebo liposome \\
\hline Mean intensity & 123 & 103.34 \\
Approximate $\mathrm{pH}$ & $<\mathrm{pH} 4.0$ & $<\mathrm{pH} 4.0$ \\
\hline
\end{tabular}

protonation increases the drug's aqueous-solubility and decreases its $\log \mathrm{D}$. When the $\mathrm{pH}$ decreases, topotecan becomes protonated and more soluble by $>100$-fold. Topotecan has only one pKa. Topotecan with an amino group can only cross the membrane in its neutral form and also required to interact with counter ion inside liposome to retain inside the liposome. Any weak base or weak acid enters inside the liposome only in its unionized form. Topotecan hydrochloride is salt of a weak base and mineral acid. So upon solubilization, it partially dissociates in and will remain in equilibrium with its ionized form and unionized form. Unionized form enters inside the liposome by crossing the lipid membrane and gets ionized with available phosphate ions. Ionization of topotecan with phosphate ion forms a gel-like structure (as depicted in Cry-TEM analysis) which locks the topotecan inside the liposome. This phenomenon will continue till all topotecan molecules get entrapped inside the liposome. This effect enables their remote loading. The result of less than $1 \%$ free drug in prepared formulation supports the argument and in line with other reported literature [29].

Topotecan has fluorescent properties, as it contains a fluorophore group. It undergoes self-quenching, which results from self-association of the drug in a given system and is dependent on the concentration of the fluorophore in the system. The objective of the study was to evaluate the physical form of topotecan inside liposomes by fluorescence spectroscopy. Topotecan hydrochloride usually gives one peak as part of emission spectra. This phenomenon is well documented. Our liposomal formulation contains topotecan bind with phosphate ions. So, topotecan phosphate might be having the property of quenching emission spectra (two peaks) as compared to significantly intensified emission (one peak) of topotecan hydrochloride which is indicative of encapsulation of topotecan inside the liposome. Placebo liposome does not contain the drug topotecan. So "no peak" is expected and actually observed in the experiment.

$\mathrm{X}$-ray diffraction (XRD) studies (small angle X-ray scattering, SAXS) have been carried out to evaluate the

Table 9 Summary of AFM analysis of topotecan liposome and placebo liposome

\begin{tabular}{ll}
\hline Product & Avg. particle size \\
\hline Topotecan liposome & Average approximate particle size $\sim 200 \mathrm{~nm}$ \\
Placebo liposome & Average approximate particle size $\sim 150 \mathrm{~nm}$ \\
\hline
\end{tabular}




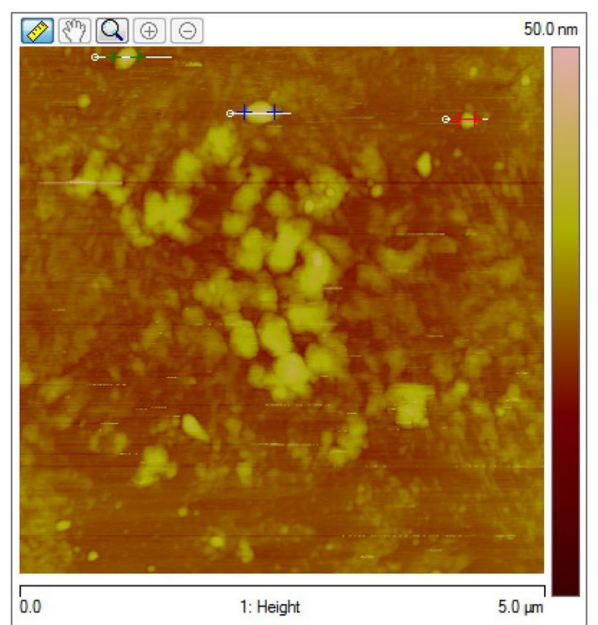

(A)

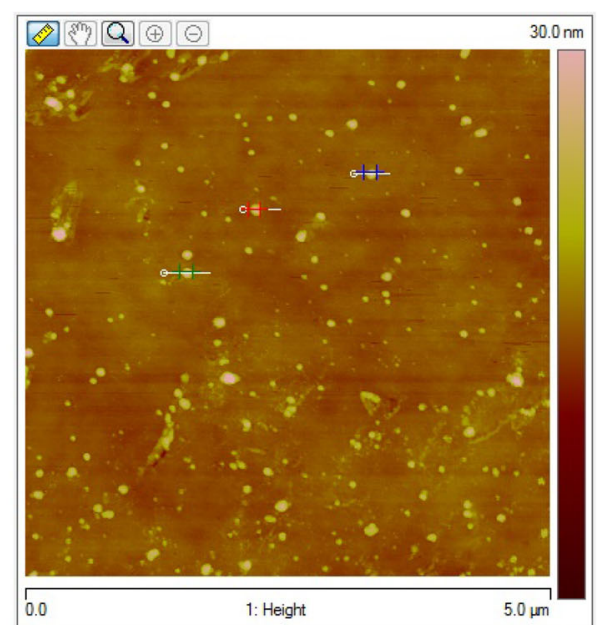

(B)

Fig. 6 AFM images of a Topotecan liposome and $\mathbf{b}$ placebo liposome

state of the encapsulated drug in the liposome. Smallangle X-ray scattering (SAXS) is an analytical technique used for the determination of the structure of solid materials. The technique gives results with accuracy and reproducibility, and additionally, the technique is nondestructive. Methodology includes irradiation of the sample with an X-ray. Angle-dependent distribution of scattered radiation (intensity) gives an idea about the structure of particle or system. This study was aiming to evaluate the physical form of topotecan inside liposomes by small-angle X-ray scattering analysis. Where due to the presence of encapsulated drugs inside the liposomes, it showed three theta peaks while placebo liposome due to the absence of topotecan did not show any theta peak. Results are indicative of the encapsulation of topotecan inside the liposome. Theta peak observed in topotecan liposome which is absent in placebo liposome indicates change in the inner environment of drug-loaded liposome.

Cryo-TEM is the technique used to get the information about the internal structure and internal environment of the liposome. In order to achieve a greater understanding of the interactions between topotecan and ammonium phosphate, this technique has been used. Liposomes are almost spherical in shape and unilamellar as revealed in Cryo-TEM analysis. The mean size of liposome observed in topotecan liposome and placebo liposome is comparable and almost below $100 \mathrm{~nm}$. The

Table 10 Glass transition temperature In DSC for topotecan liposome and placebo liposome

\begin{tabular}{ll}
\hline Product & $\begin{array}{l}\text { Temperature } \\
\text { Midpoint }\end{array}$ \\
\hline Topotecan liposome & $72.74^{\circ} \mathrm{C}$ \\
Placebo liposome & $58.88^{\circ} \mathrm{C}$ \\
\hline
\end{tabular}

topotecan drugs are observed to be present inside the aqueous compartment of the unilamellar vesicles in the form of precipitates (needle shape) while the placebo does not contain any crystalline structure inside the aqueous compartment. Observation of formation of precipitates are in-line with other researcher's observation for topotecan $[17,29]$. Topotecan crosses the bilayer and enters inside liposome in form of unionized form (topotecan base), where it might get ionized with phosphate ion and form insoluble precipitates. These formations of ionized form help topotecan to retain inside the liposome. This hypothesis is also supported in the references mentioned above.

The internal volume of topotecan liposome and placebo liposome was almost the same which indicates there was no negative impact on size morphology or inner environment after drug loading. Similarly, the internal $\mathrm{pH}$ of topotecan liposome was reported below 4 which keeps topotecan in the most suitable environment. Acidic $\mathrm{pH}$ inside the liposome help topotecan to keep in its active form throughout the circulation and till liposome reaches to tumor site.

The average particle size as observed in topotecan liposome and placebo liposome by AFM technique seems below $200 \mathrm{~nm}$ which are additional information derived during study. The images captured during AFM studies show concurrence for the morphology of particles. The atomic force microscope (AFM) study of topotecan liposome and placebo liposome shows that size representing morphology of liposome in topotecan liposome and placebo liposome are spherical in shape.

The behavior of phospholipid membranes is dependent on temperature. The transitions of this membrane from one phase to another can be identified by several techniques, using the application of temperature to the 


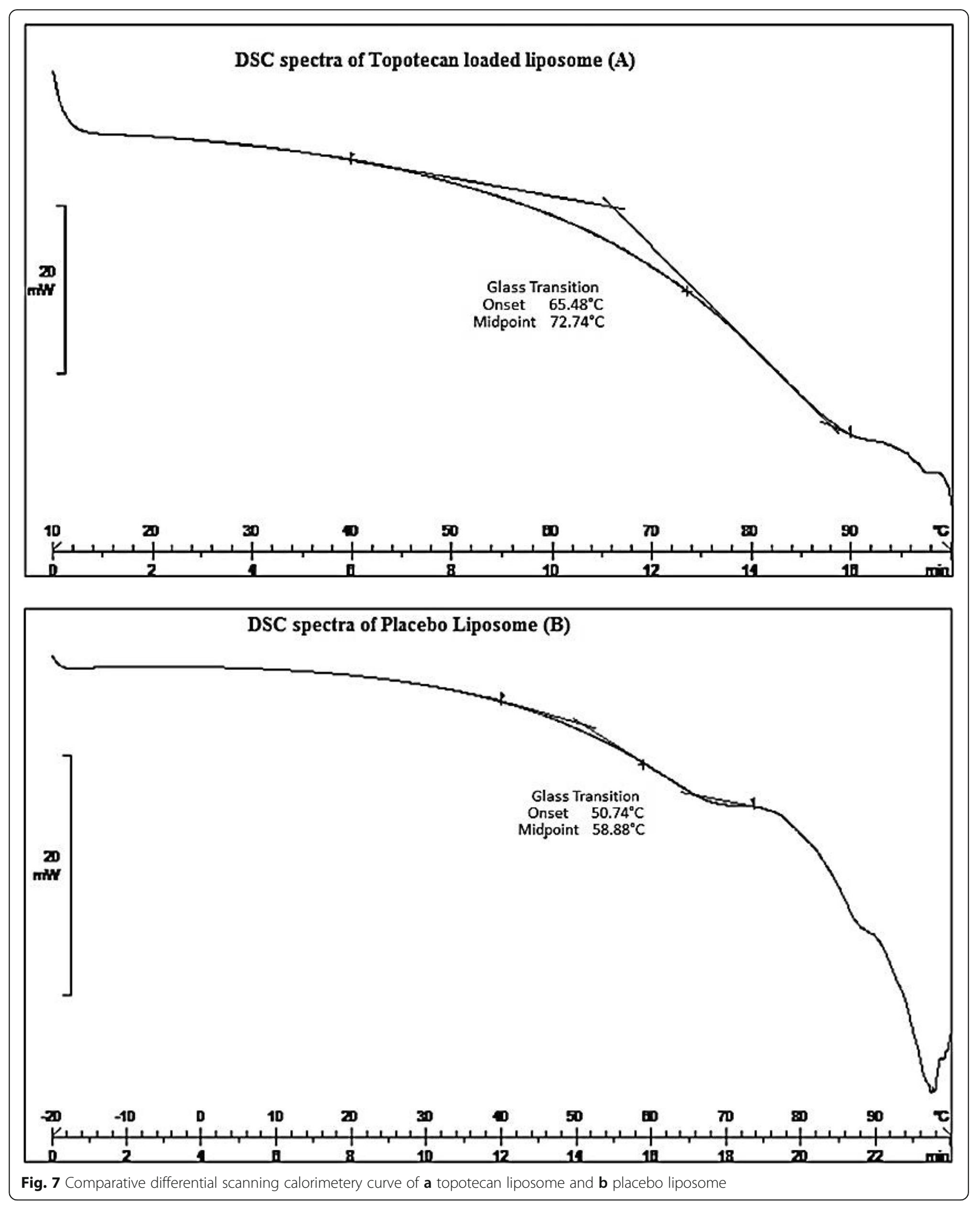

sample. The change in the phase transition is best observed at the point when the membrane converted from a tightly ordered "gel" phase to a liquid crystalline phase at increased temperatures. During the liquid-crystalline phase, a molecule can easily cross the phospholipid membrane. Liquid crystalline temperature of membranes can 
Table 11 Results of dynamic light scattering (particle size) of topotecan liposome and placebo liposome

\begin{tabular}{lll}
\hline Preparation & Placebo liposome & Topotecan liposome \\
\hline$D_{10}(n m)$ & 47.7 & 50.9 \\
$D_{50}(n m)$ & 75.7 & 77.2 \\
$D_{90}(n m)$ & 120.2 & 117 \\
PDI & 0.139 & 0.111 \\
Z-Avg (d.nm) & 75.7 & 77.2 \\
SPAN & 0.958 & 0.856 \\
\hline
\end{tabular}

be detected by various techniques like differential scanning calorimetry, thermo gravimetric analysis (TGA), and differential thermal analysis (DTA). This is an important parameter for the preparation and exploitation of liposomes vesicular structure made of phospholipid membranes. As the phase behavior of a liposomal membrane regulates the important properties like permeability, fusion, aggregation, and protein binding, it is very much essential to determine the phase transition temperature of liposomes. Lipid bilayer phase transitions will contribute to demonstrating equivalence in bilayer fluidity and uniformity. The phase transition profiles of the raw lipid excipients and liposomes should be comparable to those of the formed liposomes. Result revealed midpoint of glass transition was observed $58.88{ }^{\circ} \mathrm{C}$ for placebo liposome while $72.74{ }^{\circ} \mathrm{C}$ for topotecan-loaded liposome. These differences confirmed internal changes and encapsulation of topotecan inside the liposome. Sterically stabilized liposome of topotecan will remain intact inside the body up to temperature $50-60^{\circ} \mathrm{C}$.

Liposome size distribution is critical to ensure equivalent passive targeting. Higher particle size will increase the chance of rapid RES uptake and will result in rapid clearance from the body. Liposome size distribution measured by dynamic light scattering technique found uniform in topotecan liposome and placebo liposome. The results for $\mathrm{D}_{10}, \mathrm{D}_{50}, \mathrm{D}_{90}$, Z-Average, PDI (Polydispersity index), and SPAN for all topotecan liposomes, and placebo liposomes are comparable and indicate the uniformity of size distribution. There will not be any

Size Distribution by Intensity Topotecan Liposome (A)



Size Distribution by Intensity Placebo Liposome (B)

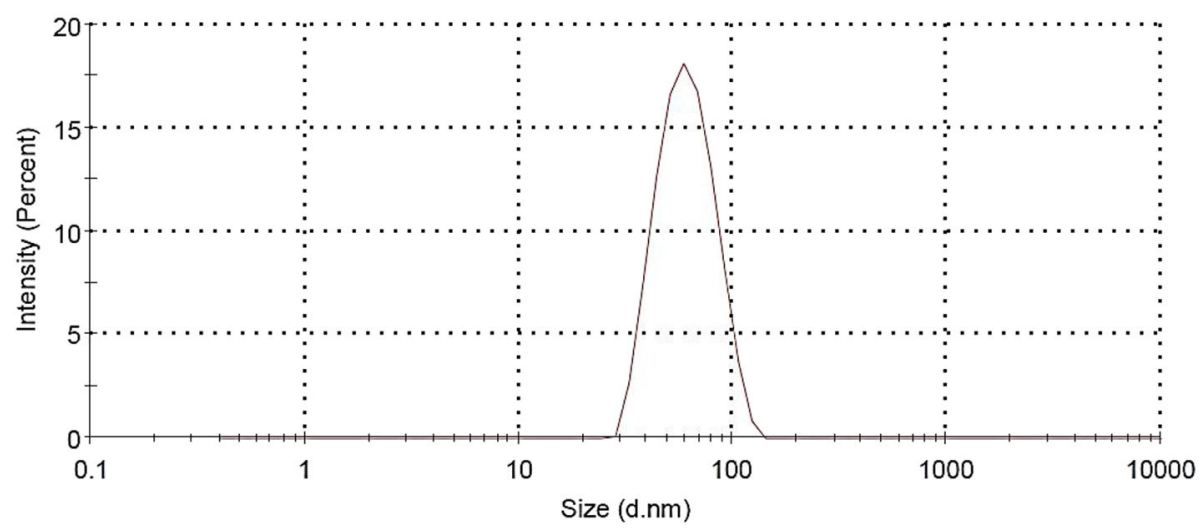

Fig. 8 Dynamic light scattering (particle size) of a topotecan liposome and $\mathbf{b}$ placebo liposome 
Table 12 Results of fixed aqueous layer thickness (FALT)

\begin{tabular}{ll}
\hline Product & Fixed aqueous layer thickness (FALT) (nm) \pm SD \\
\hline Topotecan liposome & $4.175 \pm 0.022$ \\
Placebo liposome & $3.891 \pm 0.031$
\end{tabular}

increase or decrease in size observed after drug loading inside the liposome. Results obtained for size determined by these techniques are in concurrence with that obtained by Cryo-TEM analysis.

The surface modification with methoxy polyethylene glycol (MPEG) polymer prevents the liposomes from rapid clearance by RES uptake and increases blood circulation time. The thickness of the pegylation layer should be thermodynamically limited and in several nanometers. Fixed aqueous layer thickness is different from bilayer thickness. It is the degree of pegylation around the bilayer of the liposome. As mentioned by K. SHIMADA et al., 1995 [26] pegylation prevents RES uptake of the liposome. Measurement of pegylation in quantitative term in formed liposome is quite difficult. Shimada et al. [26] provided a method to quantify the degree of pegylation in terms of PEG layer thickness using zeta potential measurement. The calculation was based on the fact that the negativity of zeta potentials of adriamycin liposomes with PEG coating decreased with increasing $\mathrm{NaCl}$ concentrations more steeply than liposome without PEG coating. If zeta potentials are measured in the presence of various concentrations of $\mathrm{NaCl}$ and plotted against $\mathrm{K}$, that is $\sqrt{\text { Concentration }} / 0.3$, the slope gives the position of the slipping plane or the thickness of the fixed aqueous layer in nm units.

The results indicate that sufficient $\mathrm{nm}$ of pegylation on the surface of liposome ensures the long circulation inside the body.

Every particle contains a net charge on the surface of the particle; it attracts the opposite charge ions to form the second layer around the first layer of ions. The layer surrounding the particle exists in two parts; first is the Stern layer which is the inner layer where the ions are strongly bound. Second is the outer layer, where ions are in diffuse and less firmly attached. Along with particle movement due to gravitational force, ions within the boundary move with it. This boundary is known as the slipping plane. The potential on the surface of this boundary is known as the zeta potential. Electrical surface charge

Table 13 Results of zeta potential

\begin{tabular}{ll}
\hline Product & Zeta potential [28] \\
\hline Topotecan liposome & -30 \\
Placebo liposome & -27 \\
\hline
\end{tabular}

on liposome can affect the clearance, tissue distribution, and cellular uptake. The zeta potential decides their state of aggregation and how they behave during processing.

The surface charge has been measured in the form of zeta potential in this investigation. The zeta potential of a liposome is directly indicative of surface charge which affects the aggregation and stability of liposome. The magnitude of the zeta potential indicates the degree of electrostatic repulsion between adjacent, similarly charged particles in a dispersion. Changes in zeta potential may imply for the aggregation, conformational changes in structure, surface modifications, and unfolding/denaturation. The results for zeta potential indicate that the effective surface charge on liposome makes them thermodynamically stable due to colloidal property. These values indicate good stability of formed liposomes.

In vitro drug leakage testing to characterize the physical state of the lipid bilayer and encapsulated topotecan should be investigated to support a lack of uncontrolled leakage under a range of physiological conditions and equivalent drug delivery to the tumor cells. To evaluate the in vivo behavior of topotecan liposome, in vitro drug leakage studies have been conducted at $37^{\circ} \mathrm{C}$ in $50 \%$ human plasma for $24 \mathrm{~h}$. The in vitro leakage of topotecan in $50 \%$ human plasma when incubated at $37^{\circ} \mathrm{C}$ was studied at $200 \mu \mathrm{g} / \mathrm{mL}$ (10 times dilution) till $24 \mathrm{~h}$. The \% leakage with $200 \mu \mathrm{g} / \mathrm{mL}$ (10 times dilution) was found to be $~ 1 \%$ till $24 \mathrm{~h}$ which indicate that liposomal formulation is having long circulation and slow-release characteristic which help them to be long-circulating inside the body and passively targeting the tumor with desired concentration.

Data from Fig. 10 show that the liposomes release topotecan very fast at $57^{\circ} \mathrm{C}$ while liposomes release topotecan slow at physiological temperature, i.e., $37^{\circ} \mathrm{C}$. Data revealed that liposomes will release drugs faster while coming in contact with cancer cells which is having a somewhat higher temperature than normal physiological conditions $\left(37^{\circ} \mathrm{C}\right)$. Similarly, the topotecan release rate was found faster in lower $\mathrm{pH}$ conditions

Table $\mathbf{1 4} \%$ Leakage of topotecan from liposome in 50\% plasma at $37^{\circ} \mathrm{C}$

\begin{tabular}{llllllll}
\hline Product & \multicolumn{7}{l}{ Time in hours (\% free drug) } \\
\cline { 2 - 8 } & $\mathbf{0}$ & $\mathbf{0 . 5}$ & $\mathbf{2}$ & $\mathbf{4}$ & $\mathbf{8}$ & $\mathbf{1 2}$ & $\mathbf{2 4}$ \\
\hline Topotecan liposome & 0.0 & 0.0 & 0.5 & 0.4 & 0.5 & 0.4 & 0.1 \\
\hline
\end{tabular}






Fig. 9 Comparative data of $\%$ leakage in $50 \%$ plasma at $37^{\circ} \mathrm{C}$ in 10 -time dilution

which is expected to be inside the tumor cell. In vitro drug release study and in vitro drug leakage in $50 \%$ human plasma study revealed that topotecan liposome is expected to remain in circulation mode in normal plasma and expected to release the drug faster when comes in contact in the tumor environment. A study was conducted according to available draft guidance by USFDA agency on similar product, i.e., doxorubicin hydrochloride liposome injection [27].

Topotecan liposomes also show desired in vitro cell toxicity which is indicative of positive response to move this formulation for further studies.

\section{Conclusion}

It is well established that for a liposome formulation to be successful, it must be able to efficiently and stably encapsulate the selected agent, have adequate drug retention attributes and release rates, have sufficient blood circulating time, and be able to resist the clearance mechanisms of the body to the target cancer cell, such that efficacy of the entrapped agent can be improved.

In this work, we attempted to address the first question using novel remote loading agent ammonium dihydrogen phosphate which gives more than $95 \%$ of encapsulation. It is also confirmed by different characterization techniques. The second and third requirements have been demonstrated using in vitro drug release study and plasma in vitro drug leakage study which shows liposome is having long circulation property due to pegylation in normal physiological condition and also having the capability to retain the drug inside the aqueous core while having faster

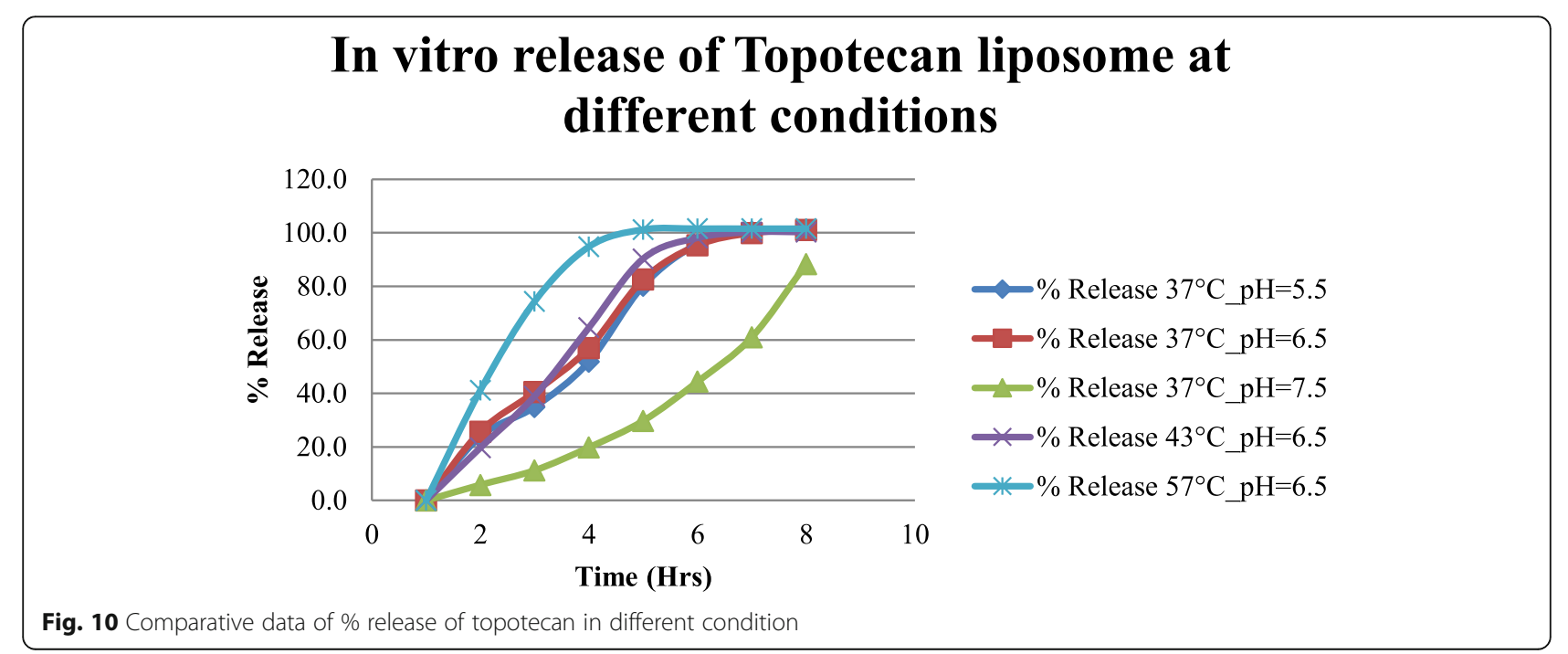




\section{Log Inhibition Vs Normalized response}

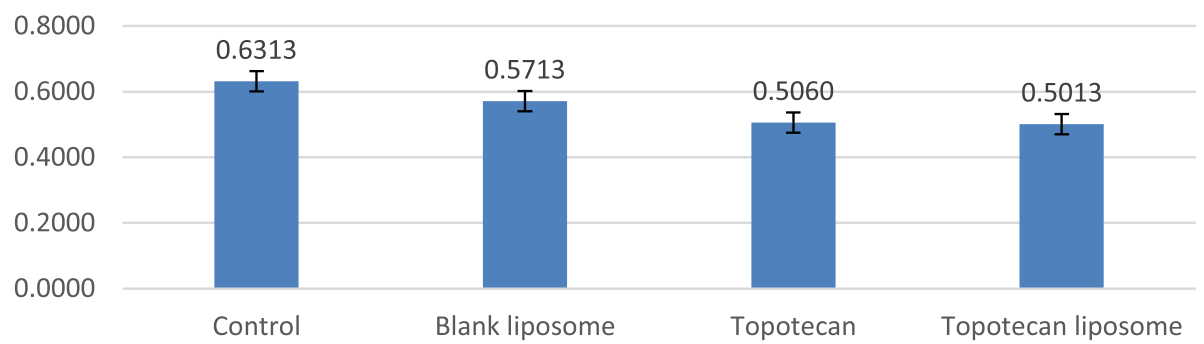

Fig. 11 Comparative log inhibition of different formulations

release rate in tumor environment such as low $\mathrm{pH}$ and high temperature. The fourth requirement is demonstrated using an in vitro cell line study (MTT assay) which indicates retention of cytotoxicity towards cancer cell in terms of efficacy of formed formulation.

In conclusion, the liposomal encapsulation improved the stability of topotecan and probably changed its distribution pattern in vivo. The inner environment of liposome has an effect on the in vitro and in vivo behavior of topotecan liposome which is characterized in this work.

\section{Abbreviations}

CPTs: Camptothecin analogs; TPT: Topotecan; HCl: Hydrochloride:

MPEG: Methoxypolyethylene glycol; MPS: Mononuclear phagocyte system; FALT: Fixed aqueous layer thickness; HPLC: High-performace chromatography; mg: Milligram; RP-HPLC: Reverse phase high-performance liquid chromatography

\section{Acknowledgements}

No acknowledgements.

\section{Authors' contributions}

NP and DP conceptualized the idea about the research work. NP was involved in planning methodology to reach the conclusion; providing personnel, environmental, and financial support and tools and instruments that are vital for the project; arranging biological materials and reagents; taking responsibility in execution of the experiments, data management and reporting; taking responsibility in logical interpretation and presentation of the results; and writing the research article.

DP was involved in constructing an idea or hypothesis for research and/or manuscript; organizing and supervising the course of the project or the article; taking the responsibility; reviewing the article before submission not only for spelling and grammar but also for its intellectual content; and guiding through the whole work and during writing of research article. All authors have read and approved the manuscript.

\section{Funding}

No funding was received.

\section{Availability of data and materials}

All data and material are available upon request.

\section{Ethics approval and consent to participate}

Not applicable

\section{Consent for publication}

Not applicable

\section{Competing interests}

The authors declare that they have no competing interests.

\section{Author details}

${ }^{1}$ Graduate School of Pharmacy-Gujarat Technological University, GTU Gandhinagar Campus, K-6 Circle, E-4 Electronic Estate, Sector-26, Gandhinagar, Gujarat 382028, India. ${ }^{2}$ Department of Pharmaceutical Sciences, Hemchandracharya North Gujarat University, Patan, Gujarat, India.

Received: 9 April 2020 Accepted: 31 August 2020

Published online: 01 November 2020

\section{References}

1. Guengerich FP (1999) Cytochrome P-450 3A4: regulation and role in drug metabolism. Annu Rev Pharmacol Toxicol 39:1-17

2. Courjault F, Leroy D, Coquery I, Toutain H (1993) Platinum complexinduced dysfunction of cultured renal proximal tubule cells. Arch Toxicol 67(5):338-346

3. Morgan MT, Carnahan MA, Immoos CE, Ribeiro AA, Finkelstein S, Lee SJ, Grinstaff MW (2003) Dendritic molecular capsules for hydrophobic compounds. J Am Chem Soc 125(50):15485-15489

4. Mueller CE (2009) Prodrug approaches for enhancing the bioavailability of drugs with low solubility. Chem Biodivers 6(11):2071-2083

5. Duncan R (2003) The dawning era of polymer therapeutics. Nat Rev Drug Discov 2(5):347

6. Torchilin VP (2005) Recent advances with liposomes as pharmaceutical carriers. Nat Rev Drug Discov 4(2):145

7. Peer D, Karp JM, Hong S, Farokhzad OC, Margalit R, Langer R (2007) Nanocarriers as an emerging platform for cancer therapy. Nat Nanotechnol 2(12):751

8. Yoo JW, Irvine DJ, Discher DE, Mitragotri S (2011) Bio-inspired, bioengineered and biomimetic drug delivery carriers. Nat Rev Drug Discov 10(7):521

9. Fox ME, Szoka FC, Fréchet JM (2009) Soluble polymer carriers for the treatment of cancer: the importance of molecular architecture. Acc Chem Res 42(8):1141-1151

10. Taggar AS, Alnajim J, Anantha M, Thomas A, Webb M, Ramsay E, Bally MB (2006) Copper-topotecan complexation mediates drug accumulation into liposomes. J Control Release 114(1):78-88

11. Broom C (1996) Clinical studies of topotecan. Ann N Y Acad Sci 803(1): $264-271$

12. Herzog TJ (2002) Update on the role of topotecan in the treatment of recurrent ovarian cancer. Oncologist 7(90005):3-10

13. Emerson DL (2000) Liposomal delivery of camptothecins. Pharmaceutical science \& technology today 3(6):205-209

14. Pantazis P, Kozielski A, Rodriguez R, Petry E, Wani M, Wall M, Giovanella B (1994) Therapeutic efficacy of camptothecin derivatives against human malignant melanoma xenografts. Melanoma Res 4(1):5-10

15. Gerrits CJ, De Jonge MJ, Schellens JH, Stoter G, Verweij J (1997) Topoisomerase I inhibitors: the relevance of prolonged exposure for present clinical development. Br J Cancer 76(7):952-962

16. Zhigaltsev IV, Maurer N, Akhong QF, Leone R, Leng E, Wang J, Semple SC, Cullis PR (2005) Liposome-encapsulated vincristine, vinblastine and vinorelbine: a comparative study of drug loading and retention. J Control Release 104(1):103-111 
17. Patankar NA, Waterhouse D, Strutt D, Anantha M, Bally MB (2013) Topophore C: a liposomal nanoparticle formulation of topotecan for treatment of ovarian cancer. Investig New Drugs 31(1):46-58

18. Forssen EA, Tökes ZA (1983) Improved therapeutic benefits of doxorubicin by entrapment in anionic liposomes. Cancer Res 43(2):546-550

19. Hong RL, Tseng YL, Chang FH (2000) Pegylated liposomal doxorubicin in treating a case of advanced hepatocellular carcinoma with severe hepatic dysfunction and pharmacokinetic study. Ann Oncol 11(3):349-354

20. Hsiang YH, Hertzberg R, Hecht S, Liu LF (1985) Camptothecin induces protein-linked DNA breaks via mammalian DNA topoisomerase I. J Biol Chem 260(27):14873-14878

21. Hertzberg RP, Caranfa MJ, Hecht SM (1989) On the mechanism of topoisomerase I inhibition by camptothecin: evidence for binding to an enzyme-DNA complex. Biochemistry 28(11):4629-4638

22. Burke TG (1996) Chemistry of the camptothecins in the bloodstream: drug stabilization and optimization of activity a. Ann N Y Acad Sci 803(1):29-31

23. Nishino M, Ashiku SK, Kocher ON, Thurer RL, Boiselle PM, Hatabu H (2006) The thymus: a comprehensive review. Radiographics 26(2):335-348

24. Cesta MF (2006) Normal structure, function, and histology of the spleen. Toxicol Pathol 34(5):455-465

25. Zucker D, Marcus D, Barenholz Y, Goldblum A (2009) Liposome drugs' loading efficiency: a working model based on loading conditions and drug's physicochemical properties. J Control Release 139(1):73-80

26. Shimada K, Miyagishima A, Sadzuka Y, Nozawa Y, Mochizuki Y, Ohshima H Hirota S (1995) Determination of the thickness of the fixed aqueous layer around polyethyleneglycol-coated liposomes. J Drug Target 3(4):283-289

27. Barenholz YC (2012) Doxil ${ }^{\circ}$-the first FDA-approved nano-drug: lessons learned. J Control Release 160(2):117-134

28. Vamvakaki V, Chaniotakis NA (2007) Pesticide detection with a liposomebased nano-biosensor. Biosens Bioelectron 22(12):2848-2853

29. Tazina EV, Kostin KV, Oborotova NA (2011) Specific features of drug encapsulation in liposomes (A review). Pharm Chem J 45(8):481-490

\section{Publisher's Note}

Springer Nature remains neutral with regard to jurisdictional claims in published maps and institutional affiliations.

\section{Submit your manuscript to a SpringerOpen ${ }^{\circ}$ journal and benefit from:}

- Convenient online submission

- Rigorous peer review

- Open access: articles freely available online

- High visibility within the field

- Retaining the copyright to your article

Submit your next manuscript at $\boldsymbol{\nabla}$ springeropen.com 